\title{
Revisión bibliográfica exploratoria sobre síndrome de apnea obstructiva del sueño y conducción profesional
}

\author{
An exploratory literature review on obstructive sleep apnea syndrome and pro- \\ fessional drivers
}

Mariholy Carolina Hernández García ', Liliana Parra Lembo ${ }^{2}$, Antonio Pérez Fernández ${ }^{3}$

1. Departamento Medicina del Trabajo. Hospital Universitario Central de Asturias. Escuela Nacional de Medicina del Trabajo. Instituto de Salud Carlos III. Madrid. España.

2. Departamento Medicina del Trabajo. Hospital Clínico Universitario San Carlos. Escuela Nacional de Medicina del Trabajo. Instituto de Salud Carlos III. Madrid. España.

3. Departamento Medicina del Trabajo. Hospital Clínico Universitario San Carlos. Escuela Nacional de Medicina del Trabajo. Instituto de Salud Carlos III. Madrid. España.

Recibido: 10-05-12

Aceptado: 25-05-12

\author{
Correspondencia: \\ Mariholy Carolina Hernández García \\ Hospital Universitario Central de Asturias \\ Servicio de Prevención de Riesgos Laborales \\ Celestino Villamil, $s / n$ \\ 33006 OVIEDO (Asturias). España. \\ Teléfono: (+34) 634982389 \\ E-mail: mariholycarolina@hotmail.com
}

Resumen

El síndrome de apnea-hipopnea del sueño es el más frecuente de los trastornos respiratorios que se producen durante el sueño, afectando alrededor del $4 \%$ de los adultos, y a casi 2 millones de personas en España. Se presenta con episodios repetidos de obstrucción de la vía aérea superior provocando un colapso del flujo de aire hacia los pulmones y síntomas como somnolencia diurna, trastornos respiratorios, cardiovasculares, psicológicos e intelectuales. La somnolencia al conducir es común entre conductores profesionales, afectando su rendimiento y la seguridad de la población. Se llevó a cabo una revisión bibliográfica exploratoria, para conocer la prevalencia de la patología en conductores profesionales, los factores del riesgo implicados, los métodos utilizados en su diagnóstico y su relación con los accidentes de tránsito. Se seleccionaron nueve bases de datos, PUBMED, IBECS, LILACS, COCHRANE LIBRARY, EMBASE, SCOPUS, SCIELO, WOS y WOK, para llevar a cabo la búsqueda entre Octubre 2011 y Enero 2012. Luego de aplicados los criterios de inclusión/exclusión se analizaron 12 artículos. La prevalencia del síndrome es similar en la mayoría de los estudios pero superior a la población general. Los factores de riesgo de mayor estudio e impacto fueron la obesidad, la somnolencia excesiva, los ronquidos. No existe consenso entre los test diagnósticos utilizados y su eficacia. Sin embargo el más utilizado ha sido la escala de Epworth (ESE). La relación siniestralidad y apnea del sueño ha sido poco estudiada en conductores profesionales.

Med Segur Trab (Internet) 2012; 59 (227) 148-167

Palabras clave: conductores profesionales, síndrome de apnea obstructiva del sueño, prevalencia, factores de riesgo. 


\section{Abstract}

The sleep apnea-hypopnea syndrome is the most common respiratory disorders that occur during sleep, affecting about $4 \%$ of adults, and nearly 2 million people in Spain. It occurs with repeated episodes of upper airway obstruction causing a flow of air collapse into the lungs and symptoms such as daytime sleepiness, respiratory, cardiovascular, psychological and intellectual disorders. Sleepiness while driving is common among professional drivers, affecting their performance and safety of the population. It was conducted an exploratory literature review to determine: the prevalence of the disease in professional drivers, the risk factors involved, the methods used in diagnosis and its relation with traffic accidents. There were selected nine databases, PubMed, IBECS, LILACS, Cochrane Library, EMBASE, Scopus, SciELO, WOS and WOK, to carry out the research between October 2011 and January 2012. After applying the criteria of inclusion / exclusion 12 articles were analyzed. The prevalence of the syndrome is similar in most studies but higher than general population. The most significant Risk factors and its impact were obesity, excessive sleepiness, snoring. There is no consensus among the diagnostic tests used and their effectiveness. However the most used is the Epworth Sleepiness Scale (ESS). The relationship between job accidents and sleep apnea has been not studied a lot in professional drivers.

Med Segur Trab (Internet) 2012; 59 (227) 148-167

Keywords: professional drivers, obstructive sleep apnea syndrome, prevalence, risk factors. 


\section{INTRODUCCIÓN}

En la actualidad el Síndrome de Apnea-Hipopnea del Sueño (SAHS) se considera como un problema de salud pública por su alta prevalencia y las evidencias de su asociación con problemas cardiovasculares, accidentes cerebrovasculares, incremento de la mortalidad y accidentes de tráfico y laborales ${ }^{1}$. Diferentes estudios epidemiológicos llevados a cabo en Estados Unidos y en Europa han evidenciado que el SAHS es una enfermedad muy prevalente que afecta entre 4-6\% de hombres y $2-4 \%$ de las mujeres en la población general adulta de edades medias ${ }^{2,3}$ y a casi 2 millones de personas en España.

El Consenso Nacional Español sobre el SAHS, define hipopnea a la objetivación de una reducción claramente discernible de la señal respiratoria $>30 \%$ y $<90 \%$, que cursa con una disminución de la saturación $>3 \%$ y/o un microdespertar en el electroencefalograma. Este mismo documento de Consenso define al SAHS como un cuadro de somnolencia excesiva, trastornos cognitivo-conductuales, respiratorios, cardíacos, metabólicos o inflamatorios secundarios a episodios repetidos de obstrucción de la vía aérea superior durante el sueño. Estos episodios se miden con el Índice de Alteración Respiratoria (IAR). Un IAR > 5 asociado a síntomas relacionados con la enfermedad y no explicados por otras causas, confirma el diagnóstico. El IAR se define como el número de apneas, hipopneas y Esfuerzos Respiratorios Asociados a Microdespertares (ERAM) por hora de sueño ${ }^{4}$.

Según un estudio de meta-análisis realizado en 2009 sobre la Apnea Obstructiva del Sueño (AOS) y el riesgo de accidente automovilístico, las personas con AOS tienen claramente un mayor riesgo de accidente; siendo esta relación un poco más fuerte para particulares que para los conductores comerciales, debido a que existen pocos estudios en conductores profesionales. Además se estima que las características que pueden predecir los accidentes en conductores con AOS incluyen el Índice de Masa Corporal (IMC), el Índice Apnea-Hipopnea (IAH), la saturación de oxígeno y, posiblemente, la somnolencia diurna ${ }^{5}$.

Recientes estudios han demostrado que no diagnosticar y por ende no tratar a los pacientes con SAHS supone un consumo de recursos 2-3 veces mayor que el de la población $\sin$ SAHS $^{6}$. En el año 2007, el número de víctimas en la carretera fue de 146.344. De estas, el $69 \%$ se produjeron de lunes a viernes, días laborables, lo que permite conjeturar que la mayor parte corresponde a personas que estaban realizando su trabajo ${ }^{7,8}$.

A pesar de estos datos sobre la relevancia del SAHS, se estima que el total de los costes atribuibles a la apnea del sueño es muy alto. Sassani y cols. han estimado que en el año 2000 hubo 800.000 colisiones que podrían ser atribuidas a apnea del sueño. El coste total fue de 15 billones de dólares y de 1.400 vidas ${ }^{9}$.

En España la detección del conductor de riesgo en el momento actual está encomendada a los Centros de Reconocimiento de Conductores (CRC). Sus facultativos, basándose en el Anexo IV del Reglamento General de Conductores ${ }^{10}$, determinan la aptitud del conductor y emiten un informe para la Jefatura Provincial de Tráfico que es la autoridad encargada de otorgar los permisos de conducción. El Síndrome de Apnea Obstructiva del Sueño (SAOS) se recoge en el apartado 7.2 sobre Trastornos del Sueño de origen respiratorio, que establece que no podrán obtener o renovar un permiso de conducción los conductores que padezcan Síndrome de Apnea Obstructiva del Sueño. No obstante estos pacientes, aportando un informe favorable de una Unidad de Sueño en el que conste que están siendo sometidos a tratamiento y control de la sintomatología diurna, podrán obtener o prorrogar permiso o licencia con un periodo máximo de vigencia de un año para los profesionales.

Sin embargo, las cifras de infradiagnóstico se estiman en un 90\%: por un lado el conductor que conoce su enfermedad con frecuencia omite esta información a la hora de realizar su reconocimiento psicofísico; a ello se añade la dificultad que encuentran los 
médicos de las Unidades de Sueño que, conscientes de tener delante un conductor de riesgo profesional, no encuentran la vía para impedir la conducción o comunicarlo a las autoridades de tráfico; dificultades que en parte derivan de la ley de protección de datos y por otra de la confidencialidad de los datos médicos y de la relación médico-paciente.

Por lo antes expuesto nos hemos propuesto la revisión de este tema, porque a pesar de tener un tratamiento efectivo en la actualidad, el interés es cada vez mayor en el ámbito de la prevención y la salud pública por el alcance del problema y la falta de políticas unánimes a nivel Europeo sobre SAOS y conducción profesional. En este orden de ideas nos hemos planteado conocer los estudios que existen en este sector laboral de alto riesgo, si existe un consenso de criterios para su diagnóstico, población más susceptible y si hay relación de causalidad con los accidentes del profesional dedicado a esta labor.

Los objetivos del presente estudio se centran en describir la prevalencia de la enfermedad, identificar aquellos factores de riesgo principalmente asociados y las pruebas diagnósticas subjetivas que con mayor frecuencia son utilizadas para valorar el riesgo y su posible relación con la siniestralidad.

\section{MATERIAL Y MÉTODOS}

Se realiza una revisión bibliográfica exploratoria de publicaciones, utilizando como términos de búsqueda la prevalencia, riesgo y diagnóstico del Síndrome de Apnea Obstructiva del Sueño (SAOS) durante el periodo comprendido entre octubre de 2011 y enero de 2012.

La búsqueda bibliográfica re realizó en las siguientes bases de datos:

- MEDLINE (Medlars Online International Literature), via PubMed.

- IBECS (Índice Bibliográfico Español en Ciencias de la Salud).

- LILACS (Literatura Latinoamericana y del Caribe en ciencias de la salud).

- Cochrane library plus (Biblioteca Cochrane plus en español).

- EMBASE (Excepta Medical data base).

- Web of Knowledge -WOK.

- Web of Science -WOS.

- SciELO España.

- Scopus.

- Google académico.

Como resultado de la búsqueda y tras aplicar un proceso de selección por pertinencia para seleccionar aquellos artículos que abordaban de forma específica los temas objeto de estudio, se obtuvo un total de 72 artículos que cumplían los criterios, de cuales 20 fueron excluidos por considerarse redundantes, quedando 52 artículos a los que se les aplicaron los criterios de inclusión y exclusión relacionados a continuación, así como un test de concordancia doble ciego con arbitraje mediante un tercer revisor en los casos de discordancia:

\section{Criterios de Inclusión}

- Conductores profesionales.

- Humanos, hombres y mujeres.

- Mayores de 18 años.

- Trabajadores activos.

- Estudios descriptivos, observacionales: transversales, caso-control y de cohorte.

- Idiomas: español e inglés.

- Texto completo.

- Artículos publicados los últimos 10 años.

- Población muestral $\geqslant 30$ conductores. 


\section{Criterios de Exclusión}

- Conductores no profesionales o no activos laboralmente.

- Estudios que valoren otros trastornos del sueño.

- Estudios que describan la somnolencia como variable independiente al SAOS.

El porcentaje de concordancia fue del $80 \%$, obteniéndose un total de 17 artículos de los cuales, no se logra obtener el documento primario de uno de ellos, quedando finalmente 16 artículos incluidos en el estudio.

Se establecieron niveles de evidencia utilizando la escala de la US Agency for Health Research and Quality ${ }^{11}$.

En la tabla I se presentan las bases de datos y el desglose sistemático de la selección de estudios utilizados. Las tablas II y III muestran la descripción de los estudios con niveles de evidencia, y los resultados obtenidos de acuerdo a los objetivos planteados, respectivamente.

Tabla 1. Bases de datos y desglose sistemático de la selección de artículos

\begin{tabular}{|c|c|c|c|c|c|}
\hline & $\begin{array}{l}\text { Términos incluidos en la } \\
\text { estrategia de búsqueda }\end{array}$ & $\begin{array}{l}\text { Número de artículos } \\
\text { seleccionados } \\
\text { por resumen } \\
\text { (abstract) }\end{array}$ & $\begin{array}{l}\text { Número de } \\
\text { artículos obtenidos } \\
\text { al retirar los } \\
\text { redundantes }\end{array}$ & $\begin{array}{c}\text { Número de artícu- } \\
\text { los que no cumplen } \\
\text { con los criterios de } \\
\text { inclusión }\end{array}$ & $\begin{array}{l}\text { Total de } \\
\text { artículos } \\
\text { utilizados }\end{array}$ \\
\hline \multirow{2}{*}{ PUBMED } & Sleep Apnea Drivers & 13 & 13 & 8 & \multirow{2}{*}{6} \\
\hline & Automobile driving & 2 & 2 & 1 & \\
\hline IBECS & $\begin{array}{l}\text { Apnea del Sueño } \\
\text { Accidentes }\end{array}$ & 1 & 1 & $\mathrm{O}$ & 1 \\
\hline LILACS & $\begin{array}{l}\text { Apnea Sueño } \\
\text { Accidentes }\end{array}$ & 2 & 2 & 1 & 1 \\
\hline $\begin{array}{l}\text { GOOGLE } \\
\text { ACADEMICO }\end{array}$ & $\begin{array}{l}\text { Apnea del Sueño } \\
\text { Conductores } \\
\text { profesionales }\end{array}$ & 7 & 7 & 4 & 4 \\
\hline & $\begin{array}{l}\text { Accidentes de Tránsito } \\
\text { Sleep Apnea }\end{array}$ & 2 & 2 & 1 & \\
\hline \multirow{3}{*}{ SCOPUS } & $\begin{array}{l}\text { Professional drivers } \\
\text { Sleep apnea syndrome } \\
\text { Automobile driving }\end{array}$ & 15 & 13 & 9 & 4 \\
\hline & $\begin{array}{l}\text { Traffic accident } \\
\text { Occupational diseases } \\
\text { Prevalence } \\
\text { Risk factors }\end{array}$ & & & & \\
\hline & Epworth sleepiness scale & 1 & 1 & 1 & 0 \\
\hline $\begin{array}{l}\text { WEB OF } \\
\text { KNOWLEDGE }\end{array}$ & $\begin{array}{l}\text { Sleep Apnea }+ \\
\text { Professional drivers } \\
\text { Public environmental } \\
\text { occupational health }\end{array}$ & 9 & 0 & 0 & 0 \\
\hline EMBASE & $\begin{array}{l}\text { Sleep Apnea syndrome } \\
\text { Professional drivers }\end{array}$ & 13 & 5 & 5 & 0 \\
\hline COCHRANE & $\begin{array}{l}\text { Sleep apnea }+ \\
\text { automobile driving } \\
\text { Sleep Apnea syndrome } \\
\text { Professional drivers }\end{array}$ & 7 & 6 & 6 & 0 \\
\hline WOS & $\begin{array}{l}\text { Sleep Apnea syndrome } \\
\text { Professional drivers }\end{array}$ & 0 & 0 & 0 & 0 \\
\hline Total & & 72 & 52 & 36 & 16 \\
\hline
\end{tabular}


Tabla II. Descripcion de los estudios con niveles de evidencia.

\begin{tabular}{|c|c|c|c|c|c|c|c|}
\hline Artículos & Autores & $\begin{array}{l}\text { Año del } \\
\text { estudio }\end{array}$ & $\begin{array}{c}\text { Año de } \\
\text { publicación }\end{array}$ & Idioma & Tipo de estudio & $\begin{array}{c}\text { Tamaño mues- } \\
\text { tral }\end{array}$ & $\begin{array}{c}\text { Nivel de } \\
\text { evidencia } \\
\text { (SIGN) }\end{array}$ \\
\hline $\begin{array}{l}\text { Síndrome de } \\
\text { Apnea Obstructiva } \\
\text { del Sueño en } \\
\text { conductores de }_{\text {camiones }^{12}}\end{array}$ & $\begin{array}{l}\text { Lucía Castro Lemos, } \\
\text { Elaine Cristina } \\
\text { Marqueze, Fernanda } \\
\text { Sashi, Gerardo } \\
\text { Lorenzi-Filho, } \\
\text { Claudia Roberta de } \\
\text { Castro Moreno }\end{array}$ & $\begin{array}{l}\text { Marzo-Julio } \\
2007\end{array}$ & 2007 & $\begin{array}{l}\text { Inglés, } \\
\text { Portugués }\end{array}$ & $\begin{array}{l}\text { Descriptivo, } \\
\text { transversal }\end{array}$ & $\begin{array}{c}209 \\
\text { conductores } \\
\text { de camiones }\end{array}$ & $\mathrm{III} / \mathrm{C}$ \\
\hline $\begin{array}{l}\text { Trastornos } \\
\text { respiratorios del } \\
\text { sueño y la calidad } \\
\text { de la vida de los } \\
\text { conductores de } \\
\text { trenes en Grecia }\end{array}$ & $\begin{array}{l}\text { Evangelina } \\
\text { Nena, Vnetia } \\
\text { Tsara, Paschalis } \\
\text { Steiropoulos, } \\
\text { Theodoros } \\
\text { Constantinidis, } \\
\text { Zoe Katsarau, } \\
\text { Pandora Christaki, } \\
\text { Demosthenes } \\
\text { Bouros }\end{array}$ & 2007 & 2008 & Inglés & $\begin{array}{c}\text { Descriptivo, } \\
\text { transversal }\end{array}$ & $\begin{array}{c}226 \\
\text { conductores }\end{array}$ & $\mathrm{III} / \mathrm{C}$ \\
\hline $\begin{array}{l}\text { Norma Nacional } \\
\text { para la evaluación } \\
\text { de la salud de } \\
\text { los trabajadores } \\
\text { de la seguridad } \\
\text { ferroviaria }^{14}\end{array}$ & $\begin{array}{l}\text { Reem Mina } \\
\text { Armand Casolin }\end{array}$ & $\begin{array}{c}\text { Febrero } \\
\text { 2004- } \\
\text { Febrero 2005 }\end{array}$ & 2007 & Inglés & $\begin{array}{l}\text { Observacional } \\
\text { Retrospectivo }\end{array}$ & $\begin{array}{c}743 \\
\text { conductores } \\
\text { de trenes } \\
283 \text { reclutas }\end{array}$ & $\mathrm{III} / \mathrm{C}$ \\
\hline $\begin{array}{l}\text { Prevalencia de } \\
\text { factores de riesgo } \\
\text { para el SAOS en } \\
\text { los conductores } \\
\text { de autobuses } \\
\text { interestatales }^{15}\end{array}$ & $\begin{array}{l}\text { Carlos Alberto } \\
\text { de Assis Viegas, } \\
\text { Haroldo Willuwett } \\
\text { de Oliveira }\end{array}$ & $\begin{array}{c}\text { Febrero- } \\
\text { Marzo } 2004\end{array}$ & 2006 & $\begin{array}{l}\text { Inglés, } \\
\text { Portugués }\end{array}$ & $\begin{array}{l}\text { Descriptivo, } \\
\text { Transversal }\end{array}$ & $\begin{array}{c}1200 \\
\text { encuestados } \\
262 \\
\text { participantes }\end{array}$ & $\mathrm{III} / \mathrm{C}$ \\
\hline $\begin{array}{l}\text { Proyección laboral } \\
\text { para la Apnea } \\
\text { Obstructiva } \\
\text { del Sueño en } \\
\text { conductores } \\
\text { comerciales }^{16}\end{array}$ & $\begin{array}{l}\text { Indira } \\
\text { Gurubhagavatula, } \\
\text { Greg Maislin, } \\
\text { Jonathan E. Nkwo, } \\
\text { Allan Pack }\end{array}$ & 2003 & 2004 & Inglés & $\begin{array}{c}\text { Descriptivo, } \\
\text { transversal }\end{array}$ & $\begin{array}{c}\text { Fase 1: } 1329 \\
\text { encuestados } \\
\text { Fase 2: } 406 \\
\text { AR (247) } \\
\text { BR (159) }\end{array}$ & $\mathrm{III} / \mathrm{C}$ \\
\hline $\begin{array}{l}\text { Prevalencia } \\
\text { del SAHS en } \\
\text { conductores } \\
\text { profesionales de } \\
\text { largo recorrido }{ }^{17}\end{array}$ & $\begin{array}{l}\text { J.R. Díaz, J. Guallar, } \\
\text { A. Arnedo, S. Oliva, } \\
\text { y J. Gala }\end{array}$ & $1997-1998$ & 2001 & Español & $\begin{array}{l}\text { Descriptivo } \\
\text { transversal, } \\
\text { Caso-control }\end{array}$ & $\begin{array}{c}163 \\
\text { conductores } \\
\text { Casos (41)- } \\
\text { Control (122) }\end{array}$ & (III/C) \\
\hline $\begin{array}{l}\text { Consenso Nacional } \\
\text { sobre Síndrome de } \\
\text { Apnea-Hipopnea } \\
\text { durante el Sueño }^{4}\end{array}$ & $\begin{array}{l}\text { Francisco Javier } \\
\text { Puertas-Cuesta } \\
\text { Gonzalo Pin- } \\
\text { Arboledas } \\
\text { Joan Santa María- } \\
\text { Cano }\end{array}$ & 2005 & 2005 & Español & $\begin{array}{l}\text { Documento } \\
\text { técnico }\end{array}$ & No aplica & IV/D \\
\hline $\begin{array}{l}\text { Somnolencia } \\
\text { Diurna Excesiva } \\
\text { en Conductores de } \\
\text { Camiones de Carga } \\
\text { de Alto Tonelaje }{ }^{18}\end{array}$ & $\begin{array}{l}\text { Salinas Mauricio } \\
\text { Riveros Alberto } \\
\text { Contreras Gustavo }\end{array}$ & 2006 & $\begin{array}{c}\text { Septiembre } \\
2008\end{array}$ & Español & $\begin{array}{l}\text { Descriptivo, } \\
\text { transversal }\end{array}$ & $\begin{array}{c}179 \\
\text { individuos }\end{array}$ & $\mathrm{III} / \mathrm{C}$ \\
\hline
\end{tabular}




\begin{tabular}{|c|c|c|c|c|c|c|c|}
\hline Artículos & Autores & $\begin{array}{l}\text { Año del } \\
\text { estudio }\end{array}$ & $\begin{array}{c}\text { Año de } \\
\text { publicación }\end{array}$ & Idioma & Tipo de estudio & $\begin{array}{l}\text { Tamaño mues- } \\
\text { tral }\end{array}$ & $\begin{array}{c}\text { Nivel de } \\
\text { evidencia } \\
\text { (SIGN) }\end{array}$ \\
\hline $\begin{array}{l}\text { Medicina del sueño } \\
\text { y profesionales del } \\
\text { transporte. Aspectos } \\
\text { médico-sociales con } \\
\text { especial referencia al } \\
\text { SAOS }^{19}\end{array}$ & $\begin{array}{l}\text { Joaquín Terán } \\
\text { Santos, Gabriel } \\
\text { Moreno y } \\
\text { Daniel O. } \\
\text { Rodenstein }\end{array}$ & 2009 & 2010 & Español & $\begin{array}{l}\text { Documento } \\
\text { técnico }\end{array}$ & No aplica & IV/D \\
\hline $\begin{array}{l}\text { Prevalencia } \\
\text { de factores de } \\
\text { riesgo para apnea } \\
\text { del sueño en } \\
\text { conductores de } \\
\text { mediana y larga } \\
\text { distancia }^{20}\end{array}$ & $\begin{array}{l}\text { Spuches, Maria } \\
\text { Lucrecia } \\
\text { Hatem Torres, } \\
\text { Silvana }\end{array}$ & $\begin{array}{l}\text { Septiembre- } \\
\text { Noviembre } \\
2007\end{array}$ & 2008 & Español & $\begin{array}{l}\text { Descriptivo, } \\
\text { transversal }\end{array}$ & $\begin{array}{l}91 \text { choferes } \\
\text { y } 34 \text { parejas } \\
\text { (todas } \\
\text { mujeres) } \\
\text { de los } \\
\text { conductores }\end{array}$ & $\mathrm{III} / \mathrm{C}$ \\
\hline $\begin{array}{l}\text { Evaluación de } \\
\text { la somnolencia } \\
\text { diurna excesiva } \\
\text { en conductores } \\
\text { profesionales con } \\
\text { sospecha de SAOS }\end{array}$ & $\begin{array}{l}\text { C Carmona Bernal; } \\
\text { F Capote Gil; G } \\
\text { Botebol Benhamou; } \\
\text { P García López; A } \\
\text { Sánchez Armengol; } \\
\text { J Castillo Gómez. }\end{array}$ & $\begin{array}{l}\text { Enero 1997- } \\
\text { Septiembre } \\
1998\end{array}$ & $\begin{array}{l}\text { Septiembre } \\
2000\end{array}$ & Español & $\begin{array}{l}\text { Descriptivo, } \\
\text { transversal }\end{array}$ & $\begin{array}{c}55 \\
\text { conductores }\end{array}$ & III/C \\
\hline $\begin{array}{l}\text { Monitoreo del sueño } \\
\text { en conductores de } \\
\text { ómnibus y camiones: } \\
\text { factor relevante a } \\
\text { considerar para } \\
\text { la renovación } \\
\text { de la licencia de } \\
\text { conducir }^{22}\end{array}$ & $\begin{array}{l}\text { Jorge Rey de Castro. } \\
\text { Edmundo Rosales- } \\
\text { Mayor }\end{array}$ & 2009 & 2010 & Español & $\begin{array}{l}\text { Documento } \\
\text { técnico }\end{array}$ & No aplica & IV/D \\
\hline $\begin{array}{l}\text { Evaluación del SAOS } \\
\text { en camioneros: un } \\
\text { enfoque para la } \\
\text { autoevaluación del } \\
\text { SAOS }^{23}\end{array}$ & $\begin{array}{l}\text { Ben Smith, } \\
\text { Barbara A. Phillips }\end{array}$ & 2010 & $\begin{array}{l}\text { Diciembre } \\
2010\end{array}$ & Inglés & $\begin{array}{l}\text { Observacional } \\
\text { analítico } \\
\text { (cohortes) }\end{array}$ & 595 & $\mathrm{IIb} / \mathrm{B}$ \\
\hline $\begin{array}{l}\text { Prevalencia y } \\
\text { correlación de } \\
\text { la mala calidad } \\
\text { del sueño y } \\
\text { somnolencia diurna } \\
\text { en conductores } \\
\text { de camiones en } \\
\text { Bélgica }^{24}\end{array}$ & $\begin{array}{l}\text { Lutgar Braeckman, } \\
\text { Rini Verpraet, } \\
\text { Marleen Van } \\
\text { Riesseghem, Dirk } \\
\text { Pevermagie, Dirk de } \\
\text { Bascquer }\end{array}$ & 2010 & 2010 & Inglés & $\begin{array}{l}\text { Descriptivo, } \\
\text { transversal- } \\
\text { Cohorte }\end{array}$ & 476 & $\begin{array}{c}\text { (III/C) y } \\
\text { (IIb/B) }\end{array}$ \\
\hline $\begin{array}{l}\text { Somnolencia diurna } \\
\text { y los accidentes } \\
\text { relacionados } \\
\text { con el sueño } \\
\text { en conductores } \\
\text { comerciales de } \\
\text { autobuses }^{25}\end{array}$ & $\begin{array}{l}\text { Marjorie Vennelle, } \\
\text { Heather M. } \\
\text { Engleman, } \\
\text { Neil J. Douglas }\end{array}$ & 2009 & Julio 2009 & Inglés & $\begin{array}{l}\text { Descriptivo, } \\
\text { Transversal }\end{array}$ & 677 & III/C \\
\hline $\begin{array}{l}\text { Conducción en } \\
\text { Europa: la necesidad } \\
\text { de una política } \\
\text { común para los } \\
\text { conductores con } \\
\text { SAOS }{ }^{26}\end{array}$ & Daniel Rodenstein & 2008 & Abril 2008 & Inglés & $\begin{array}{l}\text { Documento } \\
\text { técnico }\end{array}$ & No aplica & $\mathrm{IV} / \mathrm{C}$ \\
\hline
\end{tabular}


Tabla III. Resultados obtenidos de acuerdo a los objetivos planteados.

\begin{tabular}{|c|c|c|c|c|c|c|c|c|}
\hline Artículo & $\begin{array}{l}\text { Factores socio- } \\
\text { demográficos }\end{array}$ & $\begin{array}{c}\text { Hábitos } \\
\text { Otras patologías }\end{array}$ & $\begin{array}{c}\text { Condiciones de } \\
\text { trabajo }\end{array}$ & $\begin{array}{l}\text { Datos } \\
\text { Antropo- } \\
\text { métricos }\end{array}$ & $\begin{array}{l}\text { Datos del } \\
\text { sueño }\end{array}$ & $\begin{array}{l}\text { Pruebas } \\
\text { realizadas }\end{array}$ & Prevalencia & Siniestralidad \\
\hline $\begin{array}{l}\text { Síndrome } \\
\text { de Apnea } \\
\text { Obstructiva } \\
\text { del Sueño en } \\
\text { conductores de } \\
\text { camiones }^{11}\end{array}$ & $\begin{array}{l}\text {-Hombres } 98,6 \% \\
\text {-Edad media } 38,8 \\
+8,9 \text { años } \\
\text {-Sedentarios } 70 \%\end{array}$ & $\begin{array}{l}\text {-Alcohol } 74 \% \text { fin } \\
\text { de semana } \\
\text {-No fumadores } \\
80,1 \% \\
\text {-HTA 9,6\% }\end{array}$ & $\begin{array}{l}\text {-Empleo } \\
\text { Informal } \\
(58 \%) \\
\text { Factor protector } \\
\text {-Trabajo en } \\
\text { Distribución/ } \\
\text { Recolección } \\
-70 \% \text { horarios } \\
\text { irregulares }\end{array}$ & $\begin{array}{l}\text {-IMC } \\
\text { medio } \\
26,5+4,4 \\
\mathrm{~kg} / \mathrm{m}^{2} \\
- \text {-Sobrepeso } \\
47,8 \% \\
\text {-Obesidad } \\
15,8 \%\end{array}$ & $\begin{array}{l}\text {-Ronquidos } \\
\text { habituales } \\
38,7 \% \\
\text {-Ronquidos } \\
\text { molestos para } \\
\text { otros >2v/sem } \\
20 \% \\
\text {-Despiertan con } \\
\text { cansancio 21,5\% } \\
\text {-Dificultad para } \\
\text { conciliar el } \\
\text { sueño 17\% } \\
\text {-Quedarse } \\
\text { dormido } \\
\text { conduciendo } \\
34,5 \% \text { (25\% AR } \\
\text { de SAOS) } \\
\text {-Buena calidad } \\
\text { sueño 65\% }\end{array}$ & $\begin{array}{l}\text {-Cuestionario de } \\
\text { Actividad física } \\
\text { Internacional } \\
\text {-Cuestionario de } \\
\text { Berlín }\end{array}$ & $\begin{array}{c}11,5 \% \\
(24 \\
\text { conductores } \\
\text { con Alto } \\
\text { Riesgo de } \\
\text { SAHS) }\end{array}$ & $\begin{array}{l}15,9 \% \text { de los } \\
\text { accidentados tenían } \\
\text { Alto Riesgo de SAHS }\end{array}$ \\
\hline $\begin{array}{l}\text { Trastornos } \\
\text { respiratorios } \\
\text { del sueño y } \\
\text { la calidad de } \\
\text { la vida de los } \\
\text { conductores } \\
\text { de trenes en } \\
\text { Grecia }^{12}\end{array}$ & $\begin{array}{l}\text {-Hombres } 100 \% \\
\text { Edad media } \\
46,9+3,9\end{array}$ & -Fumadores $59,7 \%$ & & $\begin{array}{l}\text {-IMC en } \\
\text { c. con IAH } \\
<15 \\
28,3+3,2 \\
\text {-IMC en c. } \\
\text { con IAH } \\
>15 \\
33,3+4,4 \\
\text {-Ci. cuello } \\
\text { media en } \\
\text { c. con IAH } \\
<15 \\
40,2+2,4 \\
\text {-Ci. cuello } \\
\text { media en } \\
\text { c. con IAH } \\
>15 \\
43,1+2,3 \\
\text {-Ci. cintura } \\
\text { en c. con } \\
\text { IAH }<15 \\
109,4+9,4 \\
\text {-Ci. cintura } \\
\text { en c. con } \\
\text { IAH }>15 \\
113+10\end{array}$ & $\begin{array}{l}\text {-Roncadores: } \\
158(69,9 \%) \\
\text {-Roncadores } \\
\text { fuertes } \\
79(32,7 \%) \\
\text {-Ronquidos } \\
>5 \text { v/s } \\
44(19,5 \%) \\
\text {-Ronquidos } \\
\text { percibidos } \\
26(11,5 \%) \\
\text {-Ronquidos } \\
\text { percibidos por } \\
\text { las esposas } \\
41(18,1 \%) \\
\text {-Buena calidad } \\
\text { del sueño } 106 \\
\text { (46,9\%) } \\
\text {-Mala calidad } \\
\text { del sueño } \\
5(2,2 \%) \\
\text {-Sueño } \\
\text { insuficiente } \\
51(22,6 \%) \\
\text {-Media del } \\
\text { sueño } \\
6,56 \mathrm{~h}+1,44 \\
\text {-Despertares } \\
124(54,9 \%)\end{array}$ & $\begin{array}{l} \\
\\
\\
\text {-ESS } \\
\text { ESS }>10 \quad 7,1 \%(P m \\
12,9+2) \\
\text { ESS > 10> } \\
\text { promedio de edad } \\
\text {-Índice de SatO2 } \\
93,2+2,5 \% \\
\text {-SF-36 } \\
\text { No diferencias } \\
\text { significativas con } \\
\text { la población }\end{array}$ & $\begin{array}{c}- \text { IAH }<15 \\
39,79 \% \\
\text {-IAH }>15 \\
29,7+19,9 \\
\text { ev./h (11 } \\
\text { conductores) } \\
\text { IAH }>30 \text { e } \\
\text { ESS }=6 \\
2 \\
\text { conductores }\end{array}$ & \\
\hline
\end{tabular}

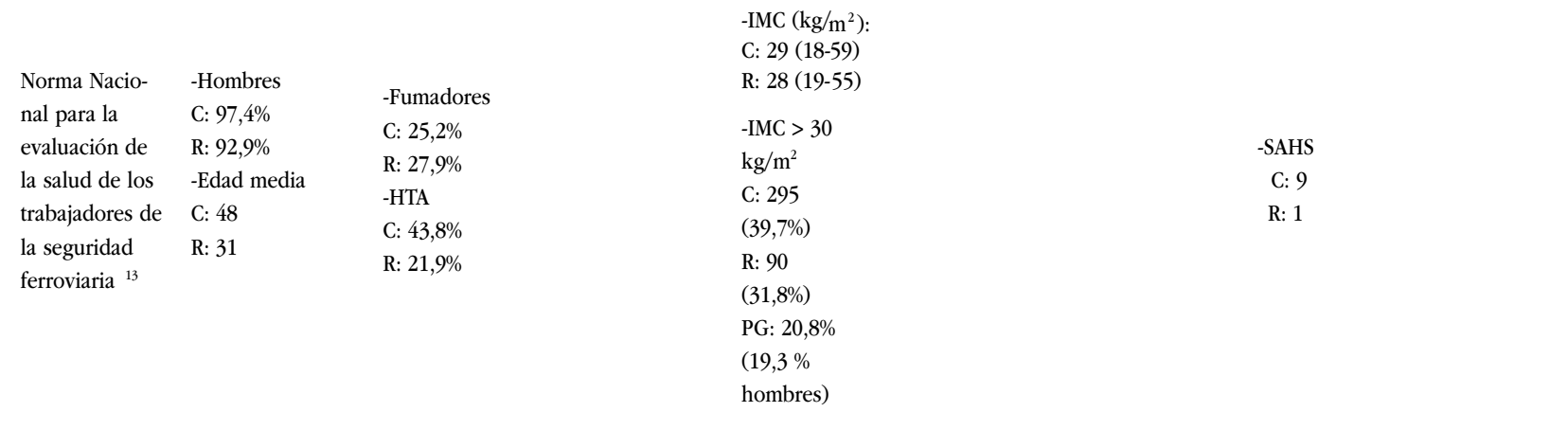




\begin{tabular}{|c|c|c|c|c|c|c|c|c|}
\hline Artículo & $\begin{array}{l}\text { Factores socio } \\
\text { demográficos }\end{array}$ & $\begin{array}{c}\text { Hábitos } \\
\text { Otras patologías }\end{array}$ & $\begin{array}{c}\text { Condiciones de } \\
\text { trabajo }\end{array}$ & $\begin{array}{l}\text { Datos } \\
\text { Antropo- } \\
\text { métricos }\end{array}$ & $\begin{array}{l}\text { Datos del } \\
\text { sueño }\end{array}$ & $\begin{array}{l}\text { Pruebas } \\
\text { realizadas }\end{array}$ & Prevalencia & Siniestralidad \\
\hline $\begin{array}{l}\text { Prevalencia } \\
\text { de factores de } \\
\text { riesgo para } \\
\text { el síndrome } \\
\text { de apnea } \\
\text { obstructiva del } \\
\text { sueño en los } \\
\text { conductores } \\
\text { de autobuses } \\
\text { interestatales }{ }^{14}\end{array}$ & $\begin{array}{l}\text {-Hombres } 100 \% \\
\text {-Edad media } 38,1 \\
+5,8(25-55)\end{array}$ & $\begin{array}{l}\text {-Fumadores } 71 \\
(27 \%) \\
\text {-C. colas } 55 \% \\
\text {-C. medicinas } 12 \% \\
\text {-C. Alcohol } 65 \% \\
\text {-C. café } 88 \%\end{array}$ & & $\begin{array}{l}\text { - Ci. } \\
\text { Cuello: } \\
40,4+2,5 \\
\text { cm (34-48) } \\
\text {-IMC media } \\
26,8+3,5 \\
(19,2-40,1) \\
\text {-Sobrepeso } \\
50 \% \\
\text {-Obesidad } \\
17,3 \%\end{array}$ & $\begin{array}{l}\text {-Ronquido } 36 \% \\
\text {-Ronquido } \\
\text { molesto para el } \\
\text { compañero } 32 \% \\
\text {-Sueño no } \\
\text { reparador } 29 \% \\
\text {-Despertares } \\
\text { con sensación } \\
\text { de ahogo } 12 \% \\
\text {-SDE } 48 \% \\
\text {-SDE e EES } \geq 10 \\
27,5 \% \\
\text {-IMC > 30 e SDE } \\
35 \%\end{array}$ & $\begin{array}{l}\text {-ESS: } \\
\text { IMC } \geq 30 \text { e SDC } \\
50 \% \text { vs IMC <30 e } \\
\text { SDC 30\% } \\
\text {-Prueba de } \\
\text { atención dividida } \\
\text { IMC } \geq 30 \text { e PAD } \\
37,3 \% \text { vs IMC }<30 \\
\text { e PAD 45,6\% } \\
\text { ESS }<10 \text { e PAD } \\
52 \% \text { vs ESS }>10 \text { e } \\
\text { PAD 37\% } \\
\text { PAD <en Ci. } \\
\text { Cuello }>42 \mathrm{~cm} \\
\text {-Prueba de } \\
\text { atención } \\
\text { sostenida }\end{array}$ & & $\begin{array}{l}7,6 \% \text { accidentes } \\
\text { atribuidos a la } \\
\text { hipesomnolencia }\end{array}$ \\
\hline
\end{tabular}

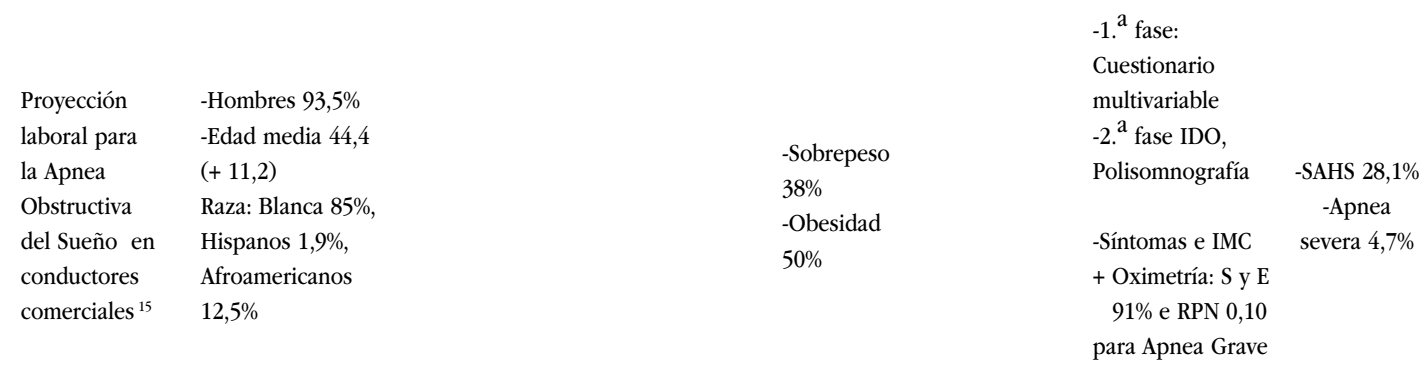

\begin{tabular}{|c|c|c|c|c|c|c|}
\hline $\begin{array}{l}\text { Prevalencia del } \\
\text { síndrome de } \\
\text { apnea-hipopnea } \\
\text { del sueño en } \\
\text { conductores } \\
\text { profesionales } \\
\text { de largo } \\
\text { recorrido }^{16}\end{array}$ & $\begin{array}{l}\text {-Hombres } 100 \% \\
\text {-Edad media } \\
40,6+4,6\end{array}$ & $\begin{array}{l}\text {-BDZ } 2 \\
\text {-HTA } \\
11(6,8 \%)\end{array}$ & $\begin{array}{l}\text {-Obesidad } \\
55(33,7 \%)\end{array}$ & $\begin{array}{l}\text {-Somnolencia } \\
\text { al conducir } \\
\text { + roncador } \\
\text { habitual + } \\
\text { pausas al } \\
\text { conducir S: } 70 \% \text {, } \\
\text { E: } 53,9 \% \text {, VPP } \\
38,6 \% \\
\text {-Somnolencia } \\
\text { al conducir } \\
\text { +roncador } \\
\text { habitual } \\
\text { S: } 67,5 \% \text {, } \\
\text { E } 62,6 \%, \text { VPP } \\
38,6 \%\end{array}$ & $\begin{array}{l}-\mathrm{IAH}<5 \quad 25,2 \% \\
\text {-IAH }>1512,3 \% \\
-\mathrm{IAH}>30 \quad 7,4 \% \\
->\text { Edad }>\mathrm{IAH} \\
\text {-Riesgos } \\
\text { asociados a } \\
\mathrm{IAH}>5: \\
\mathrm{IMC}>29 \mathrm{~kg} / \mathrm{m}^{2} \\
+ \text { somnolencia al } \\
\text { conducir }\end{array}$ & $\begin{array}{c}\text {-SAHS } \\
14(8,6 \%)\end{array}$ \\
\hline
\end{tabular}




\begin{tabular}{|c|c|c|c|c|c|c|c|c|}
\hline Artículo & $\begin{array}{l}\text { Factores socio } \\
\text { demográficos }\end{array}$ & $\begin{array}{c}\text { Hábitos } \\
\text { Otras patologías }\end{array}$ & $\begin{array}{l}\text { Condiciones de } \\
\text { trabajo }\end{array}$ & $\begin{array}{l}\text { Datos } \\
\text { Antropo- } \\
\text { métricos }\end{array}$ & $\begin{array}{l}\text { Datos del } \\
\text { sueño }\end{array}$ & $\begin{array}{l}\text { Pruebas } \\
\text { realizadas }\end{array}$ & Prevalencia & Siniestralidad \\
\hline $\begin{array}{l}\text { Somnolencia } \\
\text { diurna excesiva } \\
\text { en conductores } \\
\text { de camiones de } \\
\text { carga de alto } \\
\text { tonelaje }^{17}\end{array}$ & $\begin{array}{l}\text {-Hombres } \% \\
\text { Edad promedio: } \\
41,2\end{array}$ & -Fumadores 44,7\% & $\begin{array}{l}-75 \% \text { con } \\
>11 \text { años } \\
\text { conduciendo. } \\
\text { - Mas del 50\% } \\
\text { trabajan > } 10 \\
\text { h. día }\end{array}$ & $\begin{array}{l}\text {-Sobrepeso } \\
41 \% \\
\text { - Obesidad } \\
41 \% \\
- \text { IMC } \\
\text { normal } \\
17 \%\end{array}$ & $\begin{array}{l}\text { - Roncador } \\
>\text { del 50\% } \\
\text {-Reconocen } \\
\text { dormirse } \\
\text { conduciendo } \\
48 \%\end{array}$ & $\begin{array}{l}\text {-ESE entre } 0 \text { y } 19 . \\
-29,1 \% \text { puntaje } \\
\geq 10 \\
\text {-Individuos } \\
\text { que referían } \\
\text { haberse quedado } \\
\text { dormidos } \\
\text { conduciendo } \\
\text { presentaron } \\
\text { puntajes > altos } \\
\text { en ESE a pesar de } \\
\text { tener jornadas de } \\
\text { trabajo más cortas } \\
\text { e IMC menores. }\end{array}$ & & $\begin{array}{l}11,2 \% \text { mencionaron } \\
\text { haber tenido } \\
\text { Accidentes por } \\
\text { sueño }\end{array}$ \\
\hline $\begin{array}{l}\text { Prevalencia } \\
\text { de factores } \\
\text { de riesgo } \\
\text { para apnea } \\
\text { del sueño en } \\
\text { conductores de } \\
\text { mediana y larga } \\
\text { distancia }^{19}\end{array}$ & $\begin{array}{l}\text {-Edad media: } 46,3 \\
\text { años. }\end{array}$ & $\begin{array}{l}\text {-Fumadores según } \\
\text { encuestados: } 41 \% \\
\text {-Según parejas: } \\
\text { Fumadores } 65 \% \\
\text {-HTA: } 9 \%\end{array}$ & $\begin{array}{l}\text {-Promedio de } \\
\text { horas diarias de } \\
\text { trabajo } 10,23 \mathrm{hs} \\
\text {-Conducción de } \\
\text { noche } 42 \%\end{array}$ & $\begin{array}{l}\text {-Según las } \\
\text { parejas: } \\
\text { Sobrepeso } \\
65 \% . \\
\text {-Solo } \\
\text { el } 8 \% \\
\text { presento } \\
\text { estado } \\
\text { nutricional } \\
\text { óptimo. }\end{array}$ & $\begin{array}{l}\text {-Roncadores } \\
45 \% \\
\text {-Ahogos al } \\
\text { dormir } \\
5 \text { casos } \\
\text {->prevalencia } \\
\text { de factores } \\
\text { de riesgo en } \\
\text { roncadores. } \\
\text { (Menos la HTA) } \\
\text {-Según las } \\
\text { parejas: } \\
\text { Somnolencia } \\
\text { diurna + } \\
\text { sedentarios } 82 \%\end{array}$ & $\begin{array}{l}\text { - ESS: } \\
37 \text { conductores } \\
\text { con SDE. }\end{array}$ & & $\begin{array}{l}\text {-Accidentes en } \\
\text { conductores } \\
\text { roncadores } 37 \% \\
\text {-Accidentes en no } \\
\text { roncadores } 8 \% \\
\text {-Según las } \\
\text { convivientes: } \\
\text { accidentes de } \\
\text { tránsito en } 21 \%\end{array}$ \\
\hline $\begin{array}{l}\text { Evaluación de } \\
\text { la somnolencia } \\
\text { diurna excesiva } \\
\text { en conductores } \\
\text { profesionales } \\
\text { con sospecha de } \\
\text { SAOS }^{20}\end{array}$ & $\begin{array}{l}\text {-Edad promedio } \\
46,6 \pm 1,3\end{array}$ & & $\begin{array}{l}\text {-Todos } \\
\text { conducían más } \\
\text { de } 2 \mathrm{~h} \text { día. } \\
\text {-Tiempo medio } \\
\text { de sueño diario } \\
\text { de } 7,13 \pm 0,2 \mathrm{~h} \text {. }\end{array}$ & & $\begin{array}{l}\text {-Bajas laborales } \\
\text { por SDE: } 4 \\
\text {-IAH medio } \\
29,3 \pm 3,2 \text {. } \\
\text { - IAH medio de } \\
\text { conductores con } \\
\text { SAOS } \\
36,2 \pm 3,33 \text {. }\end{array}$ & $\begin{array}{l}\text {-ESE media: } 14,4 \\
\pm 0,6 \\
\text {-ESE } \geq 10: 85,4 \% \\
\text {-TLMS, Latencia } \\
\text { media } 4 \text { min y } \\
13 \mathrm{~s} . \\
\text {-Correlación } \\
\text { ESE/ TLMS no } \\
\text { relevante } \\
\text { (r = 0,41; } \mathrm{p}= \\
0,002 \text { ) } \\
\text {-No correlación } \\
\text { latencia media del } \\
\text { TLMS e índices } \\
\text { polisomnográfico } \\
\text {-ESE mostró } \\
\text { débil correlación } \\
\text { con el IAr } \\
\text { (r=0,26;p<0,05). }\end{array}$ & $\begin{array}{c}\text { SAOS } \\
(\mathrm{IAH}>10) \\
44 \\
\text { conductores }\end{array}$ & $\begin{array}{l}\text { - } 11 \text { referían haber } \\
\text { tenido accidentes de } \\
\text { tráfico. } \\
-5 \text { reconocían haber } \\
\text { tenido accidente de } \\
\text { tráfico por SDE. }\end{array}$ \\
\hline
\end{tabular}




\begin{tabular}{|c|c|c|c|c|c|c|c|c|}
\hline Artículo & $\begin{array}{l}\text { Factores socio } \\
\text { demográficos }\end{array}$ & $\begin{array}{c}\text { Hábitos } \\
\text { Otras patologías }\end{array}$ & $\begin{array}{l}\text { Condiciones de } \\
\text { trabajo }\end{array}$ & $\begin{array}{c}\text { Datos } \\
\text { Antropo- } \\
\text { métricos }\end{array}$ & $\begin{array}{l}\text { Datos del } \\
\text { sueño }\end{array}$ & $\begin{array}{l}\text { Pruebas } \\
\text { realizadas }\end{array}$ & Prevalencia & Siniestralidad \\
\hline $\begin{array}{l}\text { Evaluación } \\
\text { del SAOS en } \\
\text { camioneros: un } \\
\text { enfoque para la } \\
\text { autoevaluación } \\
\text { del SAOS }^{22}\end{array}$ & & -HTA 39,5\% & & $\begin{array}{l}\text {-Obesidad } \\
69.6 \% \\
\text {-IMC }>33: \\
47.6 \% \\
-\mathrm{IMC}>35 ; \\
35.6 \%\end{array}$ & $\begin{array}{l}\text {-Se dormía en } \\
\text { los semáforos } \\
20.5 \% \\
\text {-HTA lo } \\
\text { relacionó con } \\
\text { cansancio/fatiga } \\
\text { después de } \\
\text { dormir 39.5\% } \\
\text {-Apneas } \\
\text { presenciadas } \\
21 \%\end{array}$ & $\begin{array}{l}\text {-Cuestionario de } \\
\text { Berlín }\end{array}$ & & \\
\hline $\begin{array}{l}\text { Prevalencia y } \\
\text { correlación de } \\
\text { la mala calidad } \\
\text { del sueño y } \\
\text { somnolencia } \\
\text { diurna en } \\
\text { conductores de } \\
\text { camiones en } \\
\text { Bélgica }{ }^{23}\end{array}$ & $\begin{array}{l}\text {-Edad media } 42,7 \\
\text {-Educación: } \\
\text { Primaria: } 75 \\
3 \text { años de } \\
\text { secundaria: } \\
1696 \text { años de } \\
\text { secundaria: } 158 \\
\text { Bachilleres: } 67\end{array}$ & $\begin{array}{l}\text {-Fumadores } \\
219 \\
\text {-Salud } \\
\text { Pobre: } 11 \\
\text { Moderada: } 95 \\
\text { Buena: } 230 \\
\text { Muy buena: } 136\end{array}$ & $\begin{array}{l}\text { - Bajo nivel } \\
\text { de estudios } \\
\text { + fumadores } \\
\text { + horario de } \\
\text { trabajo realista > } \\
\text { riesgo de SDE. }\end{array}$ & $\begin{array}{l}\text {-IMC } \\
\text { medio } \\
27,3 \mathrm{~kg} / \mathrm{m}^{2}\end{array}$ & $\begin{array}{l}\text {-Conductores } \\
\text { que se han } \\
\text { quedado } \\
\text { dormidos por } \\
\text { la noche en la } \\
\text { carretera: } 331\end{array}$ & $\begin{array}{l}\text {-Pittsburg Sleep } \\
\text { Quality Index } \\
\text { PSQI >5 } \\
107(27,2 \%) \\
\text {-Cuestionario de } \\
\text { Berlin } \\
\text {-ESS } \\
\text { EES>10: } 84(18 \%)\end{array}$ & $\begin{array}{l}\text { Riesgo de } \\
\text { SAOS 21.5\% } \\
\quad(101)\end{array}$ & \\
\hline $\begin{array}{l}\text { Somnolencia } \\
\text { diurna y los } \\
\text { accidentes } \\
\text { relacionados } \\
\text { con el sueño } \\
\text { en conductores } \\
\text { comerciales de }^{\text {autobuses }}{ }^{24}\end{array}$ & $\begin{array}{l}\text {-Edad media } \\
42(36-51)\end{array}$ & & & $\begin{array}{l}\text {-IMC media } \\
27(24-30)\end{array}$ & $\begin{array}{l}\text {-Roncadores } \\
\text { habituales } \geq 2 \\
\text { noches / } \\
\text { semana: } 46 \\
\text {-ESE }>10 \text { y } \\
\text { roncadores } \\
\text { habituales: } 81 \\
\text {-ESE }>10 \\
\text { y apneas } \\
\text { presenciadas } \\
\text {-Conductores } \\
\text { que se han } \\
\text { quedado } \\
\text { dormidos al } \\
\text { volante } \geq 1 \text { vez/ } \\
\text { mes: } 77\end{array}$ & $\begin{array}{l}\text { ESE } \\
\text { ESE >10: } 133 \\
(20 \%)\end{array}$ & $\begin{array}{c}\text { Se estimó } \\
\text { una } \\
\text { prevalencia } \\
\text { del } 10 \%\end{array}$ & $\begin{array}{l}\text {-Conductores } \\
\text { que han tenido } \\
\text { accidentes por } \\
\text { somnolencia: } 45 \\
\text {-Conductores que } \\
\text { han estado cerca de } \\
\text { tener un accidente } \\
\text { por somnolencia: } 54\end{array}$ \\
\hline
\end{tabular}




\section{RESULTADOS}

De los 16 artículos, 12 de ellos presentaban niveles de evidencia comprendidos entre el grado II y III (1 estudio de cohortes, 1 estudio compuesto por un descriptivo transversal y uno cohortes, 1 compuesto descriptivo transversal y uno de casos-controles, 8 eran estudios descriptivos transversales y 1 retrospectivo), los otros 4 presentaban nivel de evidencia IV. A continuación se presentan los principales resultados de cada uno de ellos acuerdo a su catalogación por nivel de evidencia:

\section{Nivel evidencia IIB/B}

\subsection{Evaluación del SAOS en camioneros: un enfoque para la autoevalua- ción del SAOS ${ }^{23}$}

Ben Smith y cols. llevaron a cabo un estudio observacional analítico de cohortes, en el que en una muestra de 595 conductores profesionales se relacionó el riesgo de padecer SAOS con factores de riesgo objetivos y subjetivos. En el cuestionario de Berlín (BQ) fueron positivos 55,9\%, el 78,3\% tenían hipertensión u obesidad, el 69,6\% eran obesos, el 47,6\% tenía un IMC> $33 \mathrm{~kg} / \mathrm{m}^{2}$, y el 20,5\% informó dormirse en los semáforos. Trabajadores con síntomas clásicos de SAOS como ronquidos y somnolencia diurna excesiva, entre otros, eran más propensos a conducir con sueño; sin embargo, los datos objetivos (índice de masa corporal, historia de hipertensión) no predijeron la somnolencia durante la conducción mejor que los datos subjetivos.

\section{Nivel evidencia IIB/B - III/C}

\subsection{Prevalencia y correlación de la mala calidad del sueño y somnolencia diurna en conductores de camiones en Bélgica ${ }^{24}$}

Lutgar Braeckman y cols. llevaron a cabo un estudio en dos etapas: descriptivo transversal y de cohortes, en el que en una muestra de 476 conductores de camiones en Bélgica se estudió la prevalencia de padecer SAOS y la asociación entre factores de riesgo sociales, laborales y la somnolencia diurna excesiva (SDE). Datos de corte transversal se obtuvieron mediante un cuestionario autoadministrado que incluía el Pittsburgh Sleep Quality Index (PSQI), ESS, y BQ. La media del PSQI fue de 4.45 (2.7); la mala calidad de sueño (PSQI> 5) se encontró en el 27,2\%. La media de la ESS fue 6,79 $(4,17)$, el 18\% tenía una puntuación $>10$. El BQ indicó que el 21,5\% tenían un mayor riesgo de apnea obstructiva del sueño. La media del IMC fue de $27,3(5,1) \mathrm{kg} / \mathrm{m}^{2}$. En el análisis de regresión logística múltiple, los trabajadores con riesgo de SAOS, bajo nivel de estudios, fumadores, y un horario de trabajo realista tenían mayor riesgo de SDE. Mientras de los trabajadores con escasa experiencia laboral, insatisfacción profesional, horario de trabajo poco realista y con pobre percepción de la salud fueron relacionados con mala calidad del sueño.

\section{Nivel evidencia III/C}

\subsection{Síndrome de Apnea Obstructiva del Sueño en conductores de camiones ${ }^{12}$}

Lucia Castro Lemos y cols. en un estudio descriptivo transversal determinaron la prevalencia del SAOS e identificaron los factores asociados con un mayor riesgo entre los conductores de camiones. La población de estudio incluyó 209 conductores de camiones (edad media, 38,8 años; 98,5\% varones), en dos sucursales de una empresa de transporte. Los participantes completaron cuestionarios sobre datos sociodemográficos, la actividad física y el SAOS. La prevalencia del SAOS se estimó utilizando el Cuestionario de Berlín, 
la asociación entre el SAOS y los factores estudiados se evaluó mediante análisis de regresión univariado y multivariado. La prevalencia del SAHS en la población fue del 11,5\%. El IMC fue de 26,5 $\pm 4,4 \mathrm{~kg} / \mathrm{m}^{2}$. De los 209 conductores de camiones, $72(34,5 \%)$ reportaron haberse quedado dormido al volante y $81(38,7 \%)$ informaron sobre ronquidos. Las siguientes variables presentan una asociación estadísticamente significativa con SAOS: el empleo informal (OR $=0,27, \mathrm{p}=0,01), \mathrm{IMC} \geq 25 \mathrm{~kg} / \mathrm{m}^{2}(\mathrm{OR}=13,64, \mathrm{p}=0,01)$, y mala calidad del sueño $(O R=3,00, p=0,02)$. El $15,9 \%$ de los conductores que tuvieron accidentes tenían alto riesgo de SAOS.

\subsection{Trastornos respiratorios del sueño y la calidad de la vida de los con- ductores de trenes en Grecia ${ }^{13}$}

Evangelina Nena y cols. en un estudio descriptivo transversal evaluaron la prevalencia de trastornos respiratorios del sueño entre los conductores de trenes griegos, y su correlación con la somnolencia diurna, la calidad de vida y los síntomas. Utilizaron tres cuestionarios que fueron contestados de forma anónima por 226 conductores de trenes: un cuestionario general sobre sus características demográficas y los hábitos de sueño, la ESE y el Medical Outcomes Study 36-item forma corta (SF- 36). De los 226 conductores, 50 se sometieron a un estudio del sueño, un examen físico y una evaluación de la función respiratoria. Los participantes eran todos hombres, tenían una edad media de $46,9+3.9$ años, con IMC $28,7+3,7 \mathrm{~kg} / \mathrm{m}^{2}$, y eran fumadores $(59,7 \%)$. El ronquido fue reportado por el $69,9 \%$ de ellos y las apneas en un $11,5 \%$. La puntuación media de la ESE fue de 5,4 + 3.2. La media del IAH fue de $11+14$ eventos por hora, y el promedio de saturación de la oximetría de pulso fue $93.2+2,5 \%$. De acuerdo con la gravedad del IAH, que se dividieron en los siguientes tres grupos: grupo 1, la función normal de respiración durante el sueño ( $\mathrm{n}=19$; IAH $<5$ eventos por hora), Grupo 2, AOS leve ( $\mathrm{n}=20$; IAH, de 5,1 a $<15$ eventos por hora), grupo 3 , moderada/severa OSA ( $n=11$; IAH $>15$ eventos por hora); los tres grupos diferían en términos de IMC y perímetro de cuello, cintura y cadera, sin embargo no se detectaron diferencias en las puntuaciones de la ESE y SF-36.

\subsection{Norma nacional para la evaluación de la salud de los trabajadores de la seguridad ferroviaria: el primer año ${ }^{14}$}

Reem Mina y cols. en un estudio observacional retrospectivo determinaron la prevalencia de problemas de salud en los conductores de trenes Nueva Gales del Sur y el impacto de la nueva evaluación nacional de salud en la aptitud de maquinistas para el trabajo. Fue realizada una auditoría de los archivos de todos los conductores de trenes RailCorp (743) y recluta maquinista (283) evaluados bajo el nuevo estándar nacional para la evaluación de la salud de los trabajadores de la seguridad ferroviaria entre 2004-2005. El $25,2 \%$ de los conductores y el $27,9 \%$ de los reclutas eran fumadores, el 43,8\% de los conductores y el $21,9 \%$ de los reclutas eran hipertensos. La mediana del IMC fue $29 \mathrm{~kg} / \mathrm{m}^{2}$ (rango, $18-59 \mathrm{~kg} / \mathrm{m}^{2}$ ) para los conductores y $28 \mathrm{~kg} / \mathrm{m}^{2}$ (rango, 19-55 kg/m²) para los reclutas. La prevalencia de obesidad fue mayor tanto en reclutas como conductores al compararlo con la población masculina general. La incapacidad temporal por AOS fue dada a 9 de los conductores y 7 de los reclutas.

\subsection{Prevalencia de factores de riesgo para el síndrome de apnea obstructi- va del sueño en los conductores de autobuses interestatales ${ }^{15}$}

Carlos Alberto de Assis Viegas y cols. en un estudio descriptivo transversal determinaron la prevalencia de factores de riesgo para el SAOS en 262 conductores de autobuses interestatales. Estos fueron evaluados mediante un cuestionario diseñado para evaluar los trastornos respiratorios del sueño, la ESE, prueba de atención sostenida, prueba de la división de la atención y las medidas antropométricas. El peso corporal se encuentra por encima del ideal en el $68 \%$ de los conductores, el 34\% de los cuales tenían una circunferencia de cuello de $42 \mathrm{~cm}$. Los pilotos reportaron el uso de tabaco en un $27 \%$. La puntuación de la ESE fue de 10 puntos en el 28\%. El ronquido se informó en un 36\%, apnea del sueño en un 5\%, una sensación de asfixia durante el sueño en un $12 \%$, alteraciones del sueño en un $29 \%$ y la somnolencia durante la conducción en un $48 \%$. 
Hubo un $42 \%$ que habían estado involucrados en accidentes de tránsito, el 7,6\% de los cuales fueron atribuidos a la hipersomnolencia. La disminución de la atención dividida se correlaciona con una mayor SDE y la mayor circunferencia del cuello.

\subsection{Proyección laboral para la SAO en conductores comerciales ${ }^{16}$}

Indira Gurubhagavatula y cols. en un estudio descriptivo transversal evaluaron la polisomnografía como el criterio estándar, en comparación con precisiones de cinco estrategias para excluir la presencia de apnea del sueño severa y, en segundo lugar, cualquier apnea del sueño entre los 406 conductores de vehículos comerciales. Las estrategias utilizadas son las siguientes: (1) síntomas, (2) IMC, (3) los síntomas más el IMC, (4) un enfoque de dos etapas con síntomas más el IMC para todo el mundo, seguido por oximetría de un subconjunto; y (5) oximetría para todos. Para la exclusión de apnea severa, la estrategia en dos etapas tuvo un gran éxito, con un 91\% de sensibilidad y especificidad, y una razón de probabilidad negativa de 0,10. Esta estrategia fue similar en precisión a la oximetría, que tenía una razón de probabilidad negativa de 0,12 , y fue $88 \%$ sensible y específico del $95 \%$. Si evitamos la oximetría del todo, entonces los síntomas junto con el IMC fueron del $81 \%$ sensible y específico del $73 \%$, con una razón de probabilidad negativa de 0,26 . Por otra parte, con exclusión de cualquier apnea no se podía hacer con una precisión razonable a menos que se utilice la oximetría. Llegaron a la conclusión que la selección por dos etapas es probable que sea un medio viable de la exclusión de la apnea del sueño grave entre los conductores de vehículos comerciales.

\subsection{Prevalencia del SAHS en conductores profesionales de largo recorrido ${ }^{17}$}

J.R. Díaz y cols. en un estudio en dos etapas: descriptivo transversal y caso-control, estimaron la prevalencia de SAHS y sus factores de riesgo. Se estudiaron a 163 conductores profesionales. Se aplicó un cuestionario sobre síntomas de SAHS, factores de riesgo, examen físico y una polisomnografía. La prevalencia de conductores con IAH $>15$ fue de 19,7\% (IC del 95\%: 18,7-32,5), con SAHS de 8,6\% (IC del 95\%: 3,4-12,1). Se apreció un incremento de la prevalencia con la edad $(\mathrm{p}=0,012)$. La somnolencia al conducir o el hecho de ser roncador habitual tenían una sensibilidad del $67,5 \%$, una especificidad del $62,6 \%$ y un valor predictivo positivo del 38,6\% para detectar SAHS. En el análisis de regresión logística, los factores de riesgo fueron IMC superior a $29 \mathrm{~kg} / \mathrm{m}^{2}$ (OR: 3,56; IC del 95\%: 1,53-8,4) y la somnolencia al conducir (OR: 3,7; IC del 95\%: 1,303-10,3).

\subsection{Somnolencia Diurna Excesiva en Conductores de Camiones de Carga de Alto Tonelaje ${ }^{18}$}

Salinas Mauricio y cols. realizaron un estudio descriptivo transversal, constituido por 179 conductores de camiones de alto tonelaje, de los que se recolectó información sobre tiempos de conducción y horas de trabajo, haberse quedado dormido al volante, accidentes atribuidos al sueño y medidas antropométricas. Para medir somnolencia se utilizó la ESE. Los resultados mostraron una población de edad media de 41,2 años, todos hombres, con alta proporción de sobrepeso y obesidad del 41\%, tabaquismo 44,7\%, 75\% con más de 11 años conduciendo y más del 50\% trabaja más de 10 horas diarias. El 48\% de los conductores refiere haberse quedado dormido conduciendo y $11,2 \%$ ha tenido accidentes atribuibles a sueño. En la ESE, que se refiere a los últimos seis meses, casi un $30 \%$ de individuos presentaron puntaje de 10 o más, porcentaje que tratan de asociar a la prevalencia de SDE, sin llegar a resultados concluyentes. El análisis de asociación mostró que los conductores con mayores puntajes en ESE refieren que se han dormido conduciendo a pesar de que indican jornadas de trabajo más cortas e IMC menores. No hay relación de ESE con la edad ni tampoco con el perímetro cervical. Las largas jornadas de trabajo referidas por los conductores no tienen influencia en esta muestra. Se identificaron 13 individuos (7,3\%) obesos, roncadores y con somnolencia, los que con muy alta probabilidad tienen SAOS. 


\subsection{Prevalencia de factores de riesgo para apnea del sueño en conductores de mediana y larga distancia 20}

Spuches, María Lucrecia y cols. realizaron un estudio descriptivo transversal, constituido por 91 choferes seleccionados al azar del sindicato de conductores de larga distancia, con una edad promedio 46,3 años y por 34 parejas convivientes de dichos conductores (todas mujeres), utilizando una encuesta semi-cerrada donde se valoró edad, estado civil, nivel de instrucción, antigüedad en la tarea, trabajo nocturno, peso, talla, IMC, tabaquismo, antecedentes patológicos, roncador; características del ronquido, accidentes de tránsito, somnolencia diurna mediante la ESE. Se encontró una sobrecarga horaria con un promedio de 10,23 horas (hs.) diarias y en algunos casos 17 hs. por día. El $42 \%$ trabajaba en horario nocturno y, el $41 \%$ refirió ser fumador. Al evaluar la ESE se encontraron 37 conductores con SDE, 41 conductores roncadores (45\%) y más de 50\% presento obesidad o sobrepeso. Todos los factores de riesgos fueron más prevalentes en roncadores, menos la HTA que tuvo cifras similares. Se observó un 37\% de accidentes en roncadores frente a un $8 \%$ en no roncadores, sin poder establece relación entre estos y la apnea del sueño. Sólo 5 casos refirieron ahogos al dormir y ninguno episodios de apnea. Al evaluar las respuestas de las parejas se encontró un $65 \%$ con exceso de peso, 82\% sedentarios y con SDE, un $62 \%$ fumaba y un $21 \%$ tuvo accidentes de tránsito. Las parejas de los conductores diferenciaron las características del sueño e identificaron casos de ahogos y apneas.

\subsection{Evaluación de la somnolencia diurna excesiva en conductores profesio- nales con sospecha de SAOS ${ }^{21}$}

C Carmona Bernal y cols. realizaron un estudio descriptivo transversal en 55 conductores profesionales varones, con una edad media de 46,6 años, remitidos a la Unidad del Sueño del Hospital Virgen del Rocío de Sevilla, por sospecha de SAOS, cuya finalidad era comparar, dos métodos de medida de somnolencia diurna: uno subjetivo (ESE) y otro objetivo Test de Latencia Múltiple del Sueño (TLMS), y correlacionar ambos procedimientos con la polisomnografía. Al finalizar el estudio 11 refirieron accidentes de tráfico y 5 reconocían accidente de tráfico por SDE. Todos conducían más de $2 \mathrm{hs}$. al día. El tiempo medio de sueño diario era de 7,13 hs. El IAH medio de la serie global fue 29,3.En 44 se realizó el diagnóstico de SAOS (IAH >10). El IAH medio de los pacientes con SAOS fue de 36,2. La puntuación media de la ESE fue 14,4. El 85,4\% presentó un valor medio en la ESE $\geq$ a 10. Con respecto al TLMS, la latencia media fue $4 \min$ y $13 \mathrm{~s}$. La correlación entre la ESE y el TLMS fue significativa, más no relevante $(r=0,41 ; p=0,002)$. No hubo correlación entre la latencia media del TLMS y ningún índice polisomnográfico. La puntuación de la ESE sólo mostró una débil correlación con el Índice de Arousals (IAr), $r=0,26 ; p<0,05$. La frecuencia de SDE en esta serie es muy alta, por el TLMS (94,1\%), y la ESE $(84,5 \%)$.

\subsection{Somnolencia diurna y los accidentes relacionados con el sueño en conductores comerciales de autobuses ${ }^{25}$}

Marjorie Venelle y cols. llevaron a cabo un estudio descriptivo transversal con el fin de determinar la prevalencia de SDE y los índices de accidentes en los conductores de autobuses. Para ello se les entregó la ESE. El total de profesionales que respondieron correctamente al cuestionario fue de 677 (37\%), con una edad media de 42 años (36-51) y un IMC de 27. De ellos se dedujo que la ESE $>10$ tuvo lugar en 133 (20\%), ESE $>10$ y roncadores habituales $81(12 \%)$, ESE $>10$ y apneas presenciadas $26(4 \%)$. Los conductores que se había quedado dormidos al volante al menos 1 vez al mes fueron $77(12 \%)$, los que han padecido un accidente debido a la somnolencia fueron 45 (7\%), los que han estado cerca de tener un accidente por la SDE un 54(8\%) y 46(7\%) eran roncadores habituales, al menos 2 noches/semana. 


\section{Nivel evidencia IV/D}

\subsection{Consenso Nacional sobre Síndrome de Apnea-Hipopnea durante el SUeño ${ }^{4}$}

En este consenso se recoge toda la información obtenida por el comité del Grupo Español del Sueño (GES) con respecto a definición, diagnóstico y tratamiento del SAHS, además de exponer los riesgos de accidentes de tráfico, laborales y domésticos que puede sufrir los individuos que la padecen y sus implicaciones médico-legales, entre otros aspectos expuestos.

\subsection{Medicina del sueño y profesionales del transporte. Aspectos médico- sociales con especial referencia al SAOS ${ }^{19}$}

Joaquín Terán Santos y cols. en este artículo exponen los aspectos médico-sociales más importantes del Síndrome de Apnea Obstructiva del Sueño, planteando que en la actualidad es imprescindible incluir en los reconocimientos de los conductores y especialmente de los profesionales, cuestiones dirigidas a la identificación de trastornos del sueño, haciendo especial énfasis en la identificación del perfil del paciente con apnea del sueño y valorar en cada uno de estos los riesgos durante la conducción, además de advertirles de la necesidad de no conducir si se sienten somnolientos. También plantea la necesidad de establecer legalmente el modo de tener en cuenta el SAOS como limitador de las capacidades a la hora de obtener los permisos y licencias para conducir.

\subsection{Monitoreo del sueño en conductores de ómnibus y camiones: factor relevante a considerar para la renovación de la licencia de conducir ${ }^{22}$}

Jorge Rey de Castro y cols. en este estudio plantean una propuestas para el personal médico responsable de evaluar postulantes para renovar la licencia de conducir A-II y A-III, la cual consiste en que los conductores realicen una evaluación médica obligatoria pautada por el Ministerio del Transporte, que incluya las siguientes variables: IMC, valor de la presión arterial, configuración anatómica de la faringe de acuerdo con la clasificación de Mallampati, circunferencia del cuello, ESE y presencia de ronquido durante el sueño. En esta circunstancia el médico responsable podría aplicar una serie de criterios allí planteados con la finalidad de identificar conductores con sospecha de SAHS que requieran evaluación por especialista en trastornos del sueño quien le deberá practicar otros estudios como la polisomnografía o poligrafía respiratoria, permitiendo identificar de manera objetiva los conductores que tienen SAHS y su respectiva gravedad.

\subsection{Conducción en Europa: la necesidad de una política común para los conductores con SAOS ${ }^{26}$}

Daniel Rodenstein llevó a cabo un estudio con un comité de expertos en el que concluyeron la necesidad de la importancia de la medicina del sueño para la Salud Pública. En investigaciones médicas y científicas se había demostrado la relación entre el SAOS y los accidentes de tráfico, sin embargo el marco legal se había quedado muy atrás. La necesidad de armonización médico-legal era más que evidente.

\section{DISCUSIÓN}

Al realizar la revisión del tema en las distintas bases de datos queda demostrado el interés y la relevancia del tema a nivel médico-laboral y legal, por el gran impacto en una sociedad industrializada y maquinista en la cual la conducción profesional comprende un numeroso e importante sector. El SAHS es un problema que involucra tanto al trabajador como a terceras personas que de manera directa o indirecta pueden sufrir las consecuencias de un accidente o distracción por hipersomnolencia; en este orden de ideas dada la importancia de su conocimiento y de acuerdo a los objetivos planteados se exponen a continuación el análisis por ítems de los resultados obtenidos. 


\section{Prevalencia de SAHS}

La prevalencia del SAHS ha sido similar en todos los estudios revisados, 2 estudios han reportado cifras superiores ${ }^{16,24}$, lo cual puede estar relacionado con los test diagnósticos, ya que al usar pruebas subjetivas puede existir un infradiagnóstico, lo cual es corroborado por la polisomnografía, en otros estudios. Sin embargo la prevalencia en la población de conductores se estima que es superior a la población general, con niveles de discrepancia entre investigadores.

\section{Factores de Riesgo y SAHS}

Reconocer los factores de riesgo del SAHS es indispensable en la evaluación médica de los conductores para obtener la licencia para conducir. En 11 artículos se vincula la apnea del sueño con el IMC como uno de los principales factores involucrados en la apnea. El sobrepeso (IMC $>25 \mathrm{~kg} / \mathrm{m}^{2}$ ) y la obesidad (IMC> $30 \mathrm{~kg} / \mathrm{m}^{2}$ ) son una constante como predictores del SAHS, presentando incluso niveles superiores de obesidad que la población general en 3 de los estudios revisados; siendo la obesidad el factor de riesgo asociado con SAHS mas vinculante y estudiado.

Entre los síntomas estudiados como factores asociados a SAHS, mediante cuestionarios, se encuentran los ronquidos habituales, la SDE, la calidad del sueño. En 7 estudios se encontró que los ronquidos habituales ( $>2$ veces por semana) eran común entre los conductores de alto riesgo, en 2 de ellos se encuestaron a los acompañantes y aproximadamente entre el $20-30 \%$ reconocieron percibir los ronquidos.

La SDE es uno de los síntomas comúnmente registrados en esta patología debido a los microdespertares, el sueño no reparador, la sensación de ahogo o episodios de apnea, entre otros; siendo el SAHS la causa más frecuente. En 6 de los estudios entre 1/3 y la mitad de la población de alto riesgo encuestada refiere somnolencia durante la conducción y en 4 de los estudios entre el $25-50 \%$ de los conductores reconoce haberse dormido conduciendo. En el estudio realizado por Carlos Alberto de Assis Viegas y cols. se relaciona el IMC con la somnolencia al conducir, concluyendo que alrededor del 50\% de los que presentan un IMC $\geq 30$ tienen somnolencia diurna al conducir, en comparación con un $30 \%$ de los que presentan IMC menor de 30. Además concluye que la SDE es responsable de la gran prevalencia del consumo de drogas (café, colas) con el fin de mantener el estado de alerta durante las horas de trabajo ${ }^{15}$.

Aunque la calidad del sueño no se asocia significativamente con SAOS, los conductores que están en alto riesgo de SAOS en desarrollo clasifican la calidad del sueño como pobre, lo cual puede estar relacionado con la fragmentación resultante del incremento de microdespertares ${ }^{12}$.

Otro parámetro antropométrico utilizado en dos de los artículos hallados ha sido la circunferencia del cuello y del abdomen, Evangelina Nena y cols. relacionaron el IAH > 15 eventos/h. de la polisomnografía con una mayor circunferencia del cuello y abdomen ${ }^{13}$ y Carlos Alberto de Assis Viegas y cols. concluyeron que a mayor circunferencia del cuello menor puntuación en la prueba de atención dividida ${ }^{15}$.

Uno del los estudios asoció la situación laboral con alto riesgo del SAOS, Lucia Castro Lemos y cols. concluyeron en su estudio que el empleo informal es un factor protector, lo cual significa que la manera de organizar el trabajo puede influir en la salud de los trabajadores ${ }^{12}$.

\section{Pruebas utilizadas para el diagnóstico de SAHS}

En el SAHS se utilizan diferentes estrategias diagnósticas, donde se incluyen pruebas mediante cuestionarios realizados por los propios encuestados que permiten tener una alta sospecha de la patología y pruebas objetivas que permiten el diagnóstico de certeza. En esta revisión 7 estudios han utilizado la ESE como prueba subjetiva, la cual aplicada sin la utilización de una prueba objetiva solo permite identificar conductores con alto 
riesgo de SAHS siempre que la información suministrada tenga veracidad; donde se relaciona una escala de somnolencia $>10$ puntos como predictor de alto riesgo. En el estudio realizado por Salinas Mauricio y cols. los individuos que referían haberse quedado dormidos conduciendo presentaron puntajes más altos en ESE a pesar de tener jornadas de trabajo más cortas e IMC menores ${ }^{18}$; sin embargo otros estudios como el realizado por Carlos Alberto de Assis Viegas y cols. concluyen que existe una correlación significativa entre IMC y ESE, esta con la Prueba de Atención Dividida, y entre estos y circunferencia del cuello, indicando que a mayor IMC mayor es la hipersomnolencia, lo cual a su vez tiene una correlación negativa con la atención dividida ${ }^{15}$.

Indira Gurubhagavatula y cols. muestran en su estudio que una estrategia en dos etapas donde se combina en una primera etapa los síntomas y el IMC con la oximetría, tienen una $91 \%$ de sensibilidad y especificidad, con un cociente de probabilidad negativo, 0,10 . Este resultado es particularmente útil, porque las pruebas de confirmación como la polisomnografía es costosa y con frecuencia inaccesible, la cual se haría solo en pacientes que pasen a una segunda etapa con un alto riesgo de SAHS, ya que la estrategia prevé que la oximetría no es necesario en el $31 \%$ de la muestra, ni tampoco es necesaria la polisomnografía en el $86 \%{ }^{16}$.

Otros de los test citados han sido el Cuestionario de Berlin, el Pittsburg Sleep Quality Index, ambos han sido menos utilizados por los investigadores para medir riesgo de SAHS, teniendo una correlación débil para medir la somnolencia diurna ${ }^{12,23,24}$. A pesar de esto, J.R. Díaz y cols. concluyen que aunque los cuestionarios presentan un valor limitado al compararlos con las pruebas objetivas existe una relación entre somnolencia al conducir y ser roncador habitual, presentado una sensibilidad y especificidad de un 70 y un 53,9\% respectivamente, lo que sugiere que son de utilidad en el cribado de conductores ${ }^{17}$.

\section{Accidentes de Tráfico y siniestralidad asociados al SAHS}

La siniestralidad en relación al SAHS no está bien documentada, existe desigualdad de ideas entre si existe un mayor número de accidentes entre los conductores con SAOS que en la población general. En 4 estudios revisados al encuestar a los conductores se reconoce que alrededor de un $50 \%$ se ha quedado dormido conduciendo, sin embargo es muy difícil relacionar esto directamente con la patología, ya que existen múltiples factores implicados en la SDE, como tiempo de manejo, distancias, experiencia, toma de medicamentos y otros factores que pueden estar relacionados con el incremento de la accidentabilidad independientes a la propia patología.

\section{CONCLUSIONES}

A pesar de no poder concluir la relación entre el SAOS y los accidentes de tráfico, sería útil que todo paciente roncador con pausas respiratorias o somnolencia durante el día sea remitido a una unidad de sueño y/o un especialista para su evaluación, especialmente cuando la somnolencia dificulta la actividad laboral o social, o si hay enfermedades asociadas. La presencia de obesidad, SDE, los ronquidos, no son suficientes para remitir al paciente a una unidad de sueño; sin embargo, obliga a evaluar al paciente con más atención y a considerar un posible SAHS ${ }^{4}$.

Los estudios revisados no dan resultados concluyentes acerca de la validez de los test utilizados para evaluar los síntomas de la apnea, por la subjetividad de los mismos, los cuales pueden estar sesgados por el miedo de los trabajadores a las repercusiones laborales que implicaría el diagnóstico de SAHS.

En la actualidad los pacientes con SAHS no tratados consumen el doble de recursos que los sujetos que son tratados, debido a que estos últimos se evitan gastos indirectos en términos de absentismo, calidad de vida y accidentes de tráfico, laborales y domésticos ${ }^{22}$. Sabiendo esto lo importante es concientizar y mantener una educación continua de los conductores siendo, por lo tanto, una pieza fundamental en el manejo del riesgo, al igual 
que es necesario que las autoridades tanto políticas como sanitarias y las compañías de seguros aumenten el conocimiento y abordaje del problema para contribuir a la mejora del diagnóstico y tratamiento ${ }^{19}$.

Además debería advertirse a todos los pacientes con sospecha de apnea del sueño de los potenciales efectos de su enfermedad en la seguridad de la conducción de vehículos. Algunos de estos consejos son:

1. Reducir el tiempo de conducción a lo mínimo necesario.

2. Establecer un mínimo de tiempo de sueño.

3. Evitar el consumo de alcohol, sedantes y medicamentos productores de sueño.

4. Parar y descansar si se tiene sueño o han trascurrido 2 o más horas.

Desde el punto de vista de la prevención creemos que es necesario implementar una legislación uniforme en la Unión Europea referente a su diagnóstico, partiendo de la premisa de que el SAOS no es una enfermedad profesional, pero que los accidentes durante el trabajo, ligados a la somnolencia, son accidentes laborales y aun más en la población estudiada donde la conducción representa toda la jornada laboral.

Además hay que establecer legalmente el modo de tener en cuenta el SAOS, como limitador de las capacidades, a la hora de obtener los permisos y licencias para conducir. Una manera de empezar es responder a interrogantes como de quien es la responsabilidad de la valoración médica y las herramientas diagnósticas que se deben utilizar, cuando pueden conducir los pacientes con apnea del sueño y cada cuanto tiempo han de renovar el permiso de conducción de vehículos los pacientes con trastornos del sueño ${ }^{19}$.

\section{REFERENCIAS BIBLIOGRÁFICAS}

1. Montserrat JM, Chiner E, León A, Luque R, Maimó A, Maldonado JA. Organización asistencial, coordinación y gestión de las unidades de sueño. Relaciones con primaria y escalones asistenciales. Arch Bronconeumol 2002; 38(Supl 3):46-52.

2. Young T, Palta M, Dempsey J, et al. The occurrence of Sleep disorders breathing among middle aged adults. N Engl J Med 1993;328:1230-1236. Comentado en: Consenso Nacional sobre SAHS. Grupo Español de Sueño. Arch Bronconeumol 2005; 41(Supl 4).

3. Durán J, Esnaola S, Ramón R, Iztueta A. Obstructive sleep apnea-hypopnea and related clinical features in a popilation-based sample of subjects aged 30 to 70 years. Am J Respir Crit Care Med 2001;163:685-689. Comentado en: Consenso Nacional sobre SAHS. Grupo Español de Sueño. Arch Bronconeumol. $2005 ; 41$ (Supl 4).

4. Consenso Nacional sobre el síndrome de apneas-hipopneas del sueño. Grupo Español de Sueño (GES). Arch Bronconeumol. 2005; 41(Supl 4).

5. Tregear S, Reston J, Schoelles K, Phillips B. Obstructive sleep apnea and risk of motor vehicle crash: systematic review and meta-analysis. J Clin Sleep Med. 2009; 5(6): 573-81.

6. Peker Y, Hedner J, Johanson A, Bende M. Reduced hospitalization with cardiovascular and pulmonary disease in obstructive sleep apnea patients on nasal CPAP treatment. Sleep 1997;20:645-653.

7. Ronald J, Delaive K, Roos L, Manfreda J, Bahammam A, Kryger MH. Health care utilization in the 10 years prior to diagnosis in obstructive sleep apnea patients. Sleep 1999; 2:225-229.

8. Consejo Superior de Seguridad Vial. Observatorio Nacional de Seguridad Vial. Informe de evolución de la accidentalidad: accidentes con víctimas. Período 2003-2007. Septiembre de 2007. Disponible en: http://www.dgt.es/was6/portal/contenidos/documentos/la_dgt/recursos_humanos_empleo/ oposiciones/Accidentes_con_victimas_2003-2007 (Reunión_n_24_16-09-2008.pdf.

9. Sassani A, Findley LJ, Kryger M, Goldlust E, George C, Davidson TM. Reducing motor-vehicle collisions costs and fatalties by treating obstructive sleep apnea syndrome. Sleep 2004;27:369-80.

10. Real Decreto $772 / 1997$ de 30 de mayo por el que se aprueba el Reglamento General de Conductores.

11. Agency for Healthcare Research and Quality (sitio en internet). Disponible en: http://www.ahrq.gov. Acceso en Enero de 2012 
12. Castro L, Marqueze EC, Sachi F, Lorenzi-Filho G, de Castro CR. Obstructive sleep apnea síndrome in truck drivers. J. Bras. Pneumol. [revista en la Internet]. 2009 Jun; 35(6). Disponible en: http://www.scielo.br/ scielo.php?pid=s1806-37132009000600002\&script=sci_arttext\&tlng=en.

13. Nena E, Tsara V, Steiropoulos P, Constantinidis T, Katsarou Z, Christaki P, et al. Sleep-disordered breathing and quality of life of railway drivers in Greece. Chest [revista en la Internet]. 2008 Jul; 134(1):7986. Disponible en: http://www.ncbi.nlm.nih.gov/pubmed/18347205.

14. Mina R, Casolin A. National standard for health assessment of rail safety workers: the first year. Occupational Health and Safety [revista en la Internet]. 2007;187 (7):394-397. Disponible en: http://www. mja.com.au/public/issues/187_07_011007/min11125_fm.html.

15. De Assis CA, Willuweit H. Prevalence of risk factors for obstructive sleep apnea syndrome in interstate bus drivers. J. Bras. Pneumol. [revista en la Internet]. 2006 Mar;32(2). Disponible en: http://dx.doi. org/10.1590/S1806-37132006000200010.

16. Gurubhagavatula I, Jonathan G, Nkwuo E, Pack A. Occupational screening for obstructive sleep apnea in commercial drivers. Amer. J. Resp. Crit. Care Med. [revista en la Internet]. 2004; 170(4): 371-6. Disponible en: http://www.mendeley.com/research/occupational-screening-for-obstructive-sleep-apnea-in-commercialdrivers/.

17. Díaz JR, Guallar J, Arnedoa A, Olivab S, Galab J. Prevalencia del síndrome de apnea-hipopnea del sueño en conductores profesionales de largo recorrido. Arch Bronconeumol. [revista en la Internet]. 2001; 37(11):471-6. Disponible en: http://www.revespcardiol.org/sites/default/files/elsevier/pdf/6/6v37n11a13023053pdf001.pdf.

18. Salinas M, Riveros A, Contreras G. Excessive day drowsiness in drivers of high tonnage trucks. Ciencia \& Trabajo [revista en la Internet]. 2008 Jul-Sep;10(29). Disponible en: www.cienciaytrabajo.cl | $79 / 8179$.

19. Terán J, Moreno G, Rodenstein D. Sleep Medicine and Transport Workers. Medico-Social Aspects with Special Reference to Sleep Apnea Syndrome. Arch Bronconeumol. [revista en la Internet]. 2010; 46(3):143-7. Disponible en: http://www.archbronconeumol.org.

20. Spuches M L. Prevalencia de factores de riesgo para apnea del sueño en conductores de mediana y larga distancia. Disponible en: http://www.prevencionintegral.com/Articulos/@Datos/_ORP2008/1047.pdf.

21. Carmona C, Capote F, Botebol G, García P, Sánchez A, Castillo J. Evaluación de la somnolencia diurna excesiva en conductores profesionales con sospecha de síndrome de apnea obstructiva durante el sueño. Arch Bronconeumol. [revista en la Internet]. 2000; 36(8):436-40. Disponible en: http://www. archbronconeumol.org/bronco/ctl_servlet?_f=40\&ident=11242

22. Rey J, Rosales-Mayor E. Monitoreo del sueño en conductores de ómnibus y camiones: factor relevante a considerar para la renovación de la licencia de conducir. Rev Perú Med Exp Salud Pública [revista en la Internet]. 2010; 27(2): 260-66. Disponible en: http://www.scielo.org.pe/pdf/rins/v27n2/a16v27n2.pdf.

23. Smith B, Phillips BA. Truckers drive their own assessment for obstructive sleep apnea: a collaborative approach to online self-assessment for obstructive sleep apnea. J Clin Sleep Med. 2011;7(3) :241-245.

24. Braeckman L, Verpraet R, Van Riesseghem M, Pevermagie D, de Bascquer D. Prevalence and correlates of poor sleep quality and daytime sleepiness in belgian truck drivers. Chronobiol Int. 2011; 28(2):126-134

25. Vennelle M, Engleman H, Douglas N. Sleepiness and sleep-related accidents in commercial bus drivers. Sleep Breath 2010;14:39-42.

26. Rodenstein D. Driving in Europe: The need of a common policy for drivers with obstructive sleep apnea syndrome J. Sleep Res. 2008;17:281-284. 